\title{
Cerebrovascular reactivity measured in awake mice using diffuse correlation spectroscopy
}

\author{
Rowan O. Brothers $\odot, \stackrel{\text { a, }}{\dagger}$ Nir Atlas, ${ }^{b, \dagger}$ Kyle R. Cowdrick $\odot,{ }^{a}$ and \\ Erin M. Buckley ${ }^{\mathrm{a}, \mathrm{c}, \mathrm{d}, *}$ \\ ${ }^{a}$ Emory University and Georgia Institute of Technology, Wallace H. Coulter Department of \\ Biomedical Engineering, Atlanta, Georgia, United States \\ ${ }^{\mathrm{b}}$ Emory University and Children's Healthcare of Atlanta, Division of Critical Care Medicine, \\ Department of Pediatrics, Atlanta, Georgia, United States \\ ${ }^{c}$ Emory University School of Medicine, Department of Pediatrics, Atlanta, Georgia, \\ United States \\ ${ }^{d}$ Children's Healthcare of Atlanta, Children's Research Scholar, Atlanta, Georgia, United States
}

\begin{abstract}
Significance: Cerebrovascular reactivity (CVR), defined as the ability of the cerebral vasculature to dilate or constrict in response to a vasoactive stimulus, is an important indicator of the brain's vascular health. However, mechanisms of cerebrovascular dysregulation are poorly understood, and no effective treatment strategies for impaired CVR exist. Preclinical murine models provide an excellent platform for interrogating mechanisms underlying CVR dysregulation and determining novel therapeutics that restore impaired CVR. However, quantification of CVR in mice is challenging.
\end{abstract}

Aim: We present means of assessing CVR in awake mice using intraperitoneal injection of acetazolamide (ACZ) combined with continuous monitoring of cerebral blood flow.

Approach: Measurements of cerebral blood flow were made with a minimally invasive diffuse correlation spectroscopy sensor that was secured to an optical window glued to the intact skull. Two source-detector separations ( 3 and $4.5 \mathrm{~mm}$ ) per hemisphere were used to probe different depths. CVR was quantified as the relative increase in blood flow due to ACZ. CVR was assessed once daily for 5 days in 5 mice.

Results: We found that CVR and the response half-time were remarkably similar across hemispheres and across 3- versus 4.5-mm separations, suggesting a homogenous, whole brain response to ACZ. Mean(std) intra- and intermouse coefficients of variations were 15(9)\% and $19(10) \%$, respectively, for global CVR and $24(15) \%$ and $27(11) \%$, respectively, for global response half-time.

Conclusion: In sum, we report a repeatable method of measuring CVR in free-behaving mice which can be used to screen for impairments with disease and to track changes in CVR with therapeutic interventions.

(C) The Authors. Published by SPIE under a Creative Commons Attribution 4.0 Unported License. Distribution or reproduction of this work in whole or in part requires full attribution of the original publication, including its DOI. [DOI: 10.1117/1.NPh.8.1.015007]

Keywords: cerebrovascular reactivity; diffuse correlation spectroscopy; acetazolamide; mice; cerebral blood flow.

Paper 20065RR received Sep. 4, 2020; accepted for publication Jan. 15, 2021; published online Mar. 1, 2021.

\section{Introduction}

Cerebrovascular reactivity (CVR), defined as the ability of the cerebral vasculature to dilate or constrict in response to a vasoactive stimulus, is an important indicator of the brain's vascular

*Address all correspondence to Erin M. Buckley, erin.buckley@emory.edu

Co-first authors. 
health. ${ }^{1}$ Impaired CVR is present in numerous disease states, including cerebrovascular disease, ${ }^{2-8}$ stroke, ${ }^{9-12}$ cardiovascular disease, ${ }^{13,14}$ cardiac arrest,${ }^{15}$ traumatic brain injury, ${ }^{16-19}$ diabetes, ${ }^{20}$ and sleep apnea. ${ }^{21}$ Moreover, several studies have suggested that impaired CVR may serve as a prognostic biomarker of functional outcome. ${ }^{22-25}$ Despite mounting evidence for the relevance of CVR as a potential disease biomarker, mechanisms of cerebrovascular dysregulation are poorly understood, and effective treatment strategies for impaired CVR are lacking.

Preclinical murine models provide an excellent platform for interrogating mechanisms underlying vascular dysregulation and determining novel therapeutics that restore impaired CVR. However, quantification of CVR in mice is challenging. Common techniques to assess CVR include intravenous injection of the potent vasodilator acetazolamide (ACZ) and induction of hypercapnia via inhalation of a high-concentration (typically 5\% to 6\%) carbon dioxide gas mixture. For both of these techniques, cerebral blood flow is monitored before and after intervention, and CVR is defined as the relative percent change in blood flow from preintervention levels normalized to either the dose of $\mathrm{ACZ}$ or to the change in arterial partial pressure of carbon dioxide. Although these assessments are routinely used in humans, delivery of the vasoactive stimulus and reliable monitoring of the stimulus response is more challenging in mice. In the case of ACZ, although intravenous tail vein injection is possible, longitudinal assessment of CVR with this approach is challenging because repeated tail vein injections without the use of a catheter cause inconsistent drug delivery and increase risk of infection and vein collapse. ${ }^{26}$ Alternatively, hypercapnia requires anesthesia for gas delivery in mice, which can induce significant confounding effects on the cerebrovasculature that lead to errors in the estimation of CVR. ${ }^{27-32}$ Moreover, monitoring the amount of carbon dioxide that has reached the blood stream to accurately estimate CVR during hypercapnia requires either invasive insertion of an arterial catheter to sample blood or intubation to measure end tidal carbon dioxide concentrations. Finally, both interventions to assess CVR (ACZ/hypercapnia) require quantification of cerebral blood flow, which is non-trivial in mice due to their small size. Although a handful of techniques exist to measure $\mathrm{CBF}$ in mice, e.g., autoradiography, perfusion magnetic resonance imaging, laser Doppler flowmetry, laser speckle contrast imaging, and microultrasound, these techniques typically involve invasive surgery, anesthesia, and/or manual restraint. ${ }^{33}$ In sum, challenges in both stimulus delivery and monitoring response to the stimulus make CVR measurements in murine models technically challenging, time consuming, and preclude longitudinal assessment.

Herein, we present a novel means of assessing CVR in mice that overcomes several of these technical limitations and enables longitudinal monitoring of CVR in awake, free behaving animals. In this approach, mice are given ACZ intraperitoneally, and the cerebral blood flow response is continuously monitored with diffuse correlation spectroscopy (DCS) using a minimally invasive sensor that is secured to the intact skull. Given the relative ease of both intraperitoneal injections and assessment of blood flow with DCS, multiple longitudinal measurements of CVR can easily be acquired. In this work, we characterize average CVR in healthy mice, and we quantify the spatial and temporal heterogeneity of the CVR response.

\section{Methods}

Five 3- to 4-month-old male C57BL/6 mice were used. Measurements of resting state cerebral blood flow and CVR were assessed once daily for 5 days at the same time each day. All animal procedures were approved by Emory University Institutional Animal Care and Use Committee and followed the NIH Guidelines for the Care and Use of Laboratory Animals.

\subsection{Animal Preparation}

To enable continuous monitoring of cerebral blood flow with DCS, a laser cut clear acrylic optical window (Part No. 8560K174, McMaster-Carr) was secured to the intact skull at least 3 days prior to the start of the study. To implant the window, mice were anesthetized with $1 \%$ to $2 \%$ isoflurane, a midline incision was made that extended from between the ears to behind the eyes, fascia was removed, and a thin $(\sim 0.5 \mathrm{~mm})$ layer of dental cement (C\&B-Metabond, Parkell Inc., Edgewood, NY, USA) was employed to secure the optical window to the skull. The entire implantation procedure took $\sim 45 \mathrm{~min}$ from induction to recovery from anesthesia. 


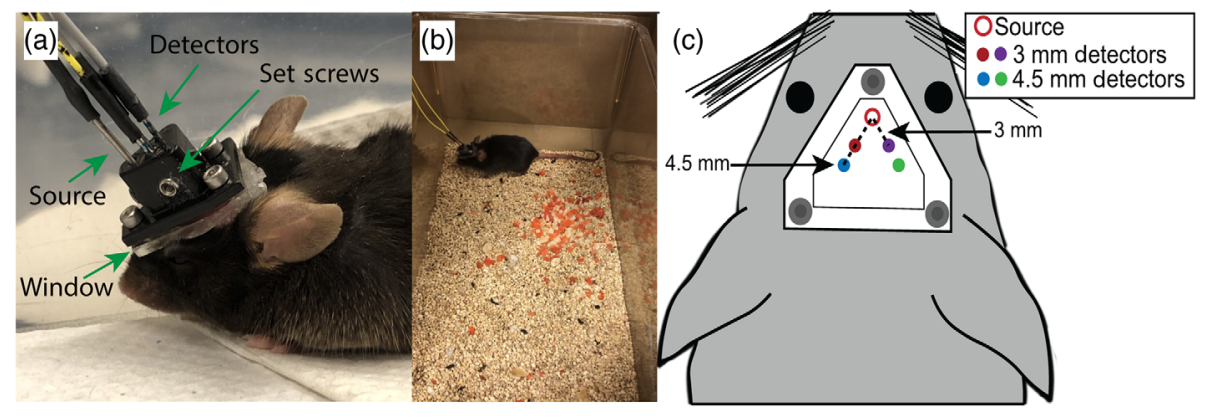

Fig. 1 Measurements of cerebral blood flow in awake mice. (a) For longitudinal measurements of cerebral blood flow, mice were briefly restrained and a custom-made, lightweight optical sensor was secured to a clear acrylic window that was attached to the intact skull. (b) Measurements were made while the animal was allowed to ambulate freely in an empty cage. (c) Diagram of the acrylic window and optical sensor positioning on the mouse head. Gray circles denote position of the screws used to secure the optical sensor to the window. One source (open circle) and four detector fibers (closed circles) were arranged within the optical sensor to enable bilateral assessment of blood flow at source-detector separations of 3 and $4.5 \mathrm{~mm}$ (Video S1, MP4, $10 \mathrm{MB}$ [URL: https:// doi.org/10.1117/1.NPh.8.1.015007.1]).

Following a 3-day recovery period, mice were acclimated to the experimental protocol by securing a custom-made, lightweight DCS sensor to the optical window. To position the sensor, mice were briefly manually restrained, and the optical sensor was secured to the optical window with three screws [Fig. 1(a)]. Once secured, mice were placed in an empty cage and allowed to ambulate freely for 10 to 20 min [Fig. 1(b) and Video S1], at which time the sensor was removed and the mice were returned to their cage. This acclimation process was repeated once daily for 3 days.

\subsection{Assessment of Cerebral Blood Flow and Cerebrovascular Reactivity}

Measurements of cerebral blood flow were made with DCS. ${ }^{34-36}$ In DCS, a coherent near-infrared source is used to illuminate the tissue surface. A detector is placed at a fixed distance from the source to detect light that has multiply-scattered through the tissue. Scattering off moving red blood cells causes the detected light intensity to fluctuate with time. Correlation diffusion theory is used to relate these intensity fluctuations to blood flow index (BFI) within the underlying tissue. Although the units of BFI $\left(\mathrm{mm}^{2} / \mathrm{s}\right)$ are not the traditional units of flow $(\mathrm{mL} / \mathrm{min} / 100 \mathrm{~g})$, numerous validation studies, ${ }^{37}$ including a recent study in mice, ${ }^{33}$ have shown that BFI is correlated with blood flow measured by other perfusion modalities, including laser Doppler flowmetry, ${ }^{38,39}$ color Doppler ultrasound, and transcranial Doppler ultrasound, ${ }^{38,40-43}$ perfusion magnetic resonance imaging, ${ }^{33,38,41,44,45}$ Xenon-CT, ${ }^{46}$ near-infrared spectroscopy, ${ }^{47}$ and fluorescent microspheres. ${ }^{48}$

The DCS instrument employed herein consisted of an 852-nm long coherence-length laser (iBeam smart, TOPTICA Photonics, Farmington, NY), an array of four photon counting avalanche photodiodes (SPCM-AQ4C-IO, Perkin-Elmer, Quebec, Canada), and a hardware autocorrelator board (Flex05-8ch, ${ }^{49} \mathrm{NJ}$ ). ${ }^{33,50}$ The animal interface for the device consisted of a custom 3D printed optical sensor with a $400-\mu \mathrm{m}$ multimode source fiber (FT-400-EMT, Thorlabs Inc., Newton, NJ, USA) and four single-mode detector fibers (780HP, Thorlabs Inc., Newton, NJ, USA) spaced 3 and $4.5 \mathrm{~mm}$ from the source [Figs. 1(a) and 1(c)]. These separations were chosen to ensure sensitivity to cortical tissue. ${ }^{51}$ Set screws on the sides of the sensor were used to secure all fibers tightly in place [Fig. 1(a)]. Data were acquired from all detectors simultaneously at a rate of $1 \mathrm{~Hz}$.

The measured intensity autocorrelation function at each time $t$ and source-detector separation $r$ was fit to the semi-infinite homogeneous solution to the correlation diffusion equation for a $\mathrm{BFI}\left[(r, t), \mathrm{mm}^{2} / \mathrm{s}\right] .^{34}$ To increase sensitivity to the decay of these measured curves, fits were restricted to autocorrelation values $>1.05$. Optical properties were assumed uniform across all mice; absorption and reduced scattering coefficients at $852 \mathrm{~nm}$ were fixed at 0.25 and $9.4 / \mathrm{cm}$, respectively. ${ }^{51}$ 


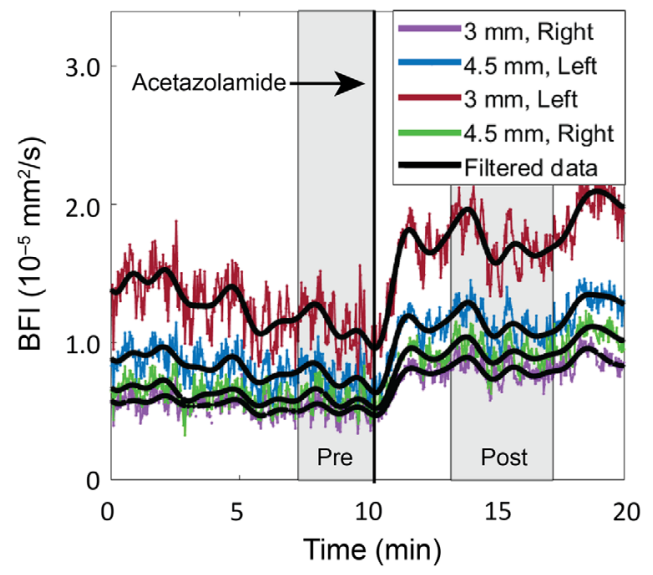

Fig. 2 Representative response to Acetazolamide (ACZ). Sample time series of the measured blood flow index $(\mathrm{BFI})$ at the four source-detector separations (colored lines), along with overlaid low-pass filtered time series used for quantification of CVR (black lines). ACZ was given after a 10-min baseline period. Gray shaded rectangles denote averaging windows for the pre- and postinjection periods used for quantification of CVR, which was quantified as the relative percent change in blood flow from pre- to post-ACZ injection.

To assess CVR, the DCS sensor was secured to the optical window, and the mice were placed in an empty cage. Blood flow was monitored continuously $(1 \mathrm{~Hz})$ throughout the duration of the experiment. After a 10-min baseline period, mice were given an intraperitoneal injection of $90 \mathrm{mg} / \mathrm{kg}$ ACZ (Sigma-Aldrich, St. Louis, MO). ACZ was dissolved in a phosphate-buffered saline solution (Lonza Walkersville Inc., MD), $\mathrm{pH} 8.8$, to yield a 32-mg/mL stock solution. Preliminary results suggested the hemodynamic effects of ACZ peak within 2 to 10 min postinjection and persist for 1 to $2 \mathrm{~h}$ (see Fig. S2 in the Supplemental Material). Thus to minimize experiment duration, blood flow was monitored for 10-min post-ACZ injection, after which time the optical sensor was removed, and the animal was returned to its cage.

To quantify CVR, BFI $(r, t)$ was first low-pass filtered at $0.005 \mathrm{~Hz}$. CVR for each sourcedetector pair was defined as

$$
\operatorname{CVR}(r)=\frac{\langle\operatorname{BFI}(r, t)\rangle_{\text {post }}-\langle\operatorname{BFI}(r, t)\rangle_{\text {pre }}}{\langle\operatorname{BFI}(r, t)\rangle_{\text {pre }}} \times 100 \%
$$

Here $\langle\operatorname{BFI}(r, t)\rangle_{\text {pre }}$ denotes a 3-min temporal mean of the filtered $\operatorname{BFI}(r, t)$ data immediately prior to injection, and $\langle\operatorname{BFI}(r, t)\rangle_{\text {post }}$ denotes a 4-min temporal mean of the filtered $\operatorname{BFI}(r, t)$ data that began 2 min after ACZ injection (Fig. 2). We also characterized the response half-time $t_{1 / 2}$ defined as the time it took the filtered $\operatorname{BFI}(r, t)$ data to reach half of the peak response postinjection, i.e., when $\operatorname{BFI}(r, t)=\frac{1}{2}\langle\operatorname{BFI}(r, t)\rangle_{\text {pre }}+\frac{1}{2}\langle\operatorname{BFI}(r, t)\rangle_{\text {post }}$.

\subsection{Statistical Analysis}

Paired Wilcoxon signed-rank tests were used to determine whether baseline BFI, CVR, and $t_{1 / 2}$ were different between hemispheres (right versus left) and between source-detector separations ( 3 versus $4.5 \mathrm{~mm}$ ). Further, linear regression analysis was used to quantify the relationship of BFI, $\mathrm{CVR}$, and $t_{1 / 2}$ across source-detector separations and hemispheres. Results are reported as slope and intercept [with associated confidence intervals (CI)], along with the coefficient of determination, $R^{2}$. Global estimates of baseline BFI, CVR, and $t_{1 / 2}$ were calculated by averaging each measured parameter across all source-detector pairs. To assess the amount of variation between mice, we first calculated the mean and standard deviation of each measured global parameter across all mice on each day, allowing us to calculate a daily intermouse coefficient of variation $\left(\mathrm{COV}_{\text {byday }}\right.$, standard deviation/mean of parameter $X$ ). We then estimated a mean(std) intermouse COV using the $\mathrm{COV}_{\text {byday }}$ values (standard deviation/mean of $\mathrm{COV}_{\text {byday }}$ ). Similarly, to assess the amount of 
variation for a given mouse across days, we first calculated the mean and standard deviation of each measured global parameter for each mouse across all days, allowing us to calculate a COV per mouse $\left(\mathrm{COV}_{\text {bymouse }}\right.$, standard deviation/mean of parameter $\left.X\right)$. We then estimated a mean(std) intramouse $\mathrm{COV}$ using the $\mathrm{COV}_{\text {bymouse }}$ values (standard deviation/mean of $\mathrm{COV}_{\text {bymouse }}$ ). All statistical analyses were performed in MATLAB ${ }^{52}$ Hypotheses tests and associated $p$-values were two-sided. Statistical significance was declared for $p$-values $<0.05$.

\section{Results}

A total of 25 measurements were made in 5 mice across 5 consecutive days. Five measurements were discarded: two were discarded due to abrupt movement during intraperitoneal injections, and three were discarded in mouse 4 due to poor sensor contact with the optical window as a result of dried dental cement on the window surface.

Resting state blood flow showed significant temporal variation over the 10-min baseline period, as seen in the representative timeseries in Fig. 2. This temporal variability was not thought to be due to motion artifact, as it was observed even when the animal was at rest.

Across all mice, baseline BFI at the 3-mm source-detector separation $\left(\mathrm{BFI}_{3} \mathrm{~mm}\right)$ was systematically higher than BFI at the $4.5-\mathrm{mm}$ separation $\left(\mathrm{BFI}_{4.5 \mathrm{~mm}}\right)$ for both the right and left hemispheres $(p<0.001$ and $p=0.02$, respectively). At the 3-mm separation, baseline BFI on the left hemisphere $\left(\mathrm{BFI}_{\text {left }}\right)$ trended higher than $\mathrm{BFI}$ on the right hemisphere $\left(\mathrm{BFI}_{\text {right }}, p=0.06\right)$, whereas at the $4.5-\mathrm{mm}$ separation, $\mathrm{BFI}_{\text {right }}$ trended higher than $\mathrm{BFI}_{\text {left }}(p=0.08)$. Baseline $\mathrm{BFI}_{3 \mathrm{~mm}}$ was weakly correlated with $\mathrm{BFI}_{4.5 \mathrm{~mm}}$ on the left hemisphere [Fig. 3(a), Table 1] and strongly correlated with $\mathrm{BFI}_{4.5 \mathrm{~mm}}$ on the right hemisphere [Fig. 3(b), Table 1]. Similarly, $\mathrm{BFI}_{\text {right }}$ and $\mathrm{BFI}_{\text {left }}$ were significantly correlated at the 3-mm separation [Fig. 3(c), Table 1] and at the 4.5-mm separation [Fig. 3(d), Table 1]. Intramouse COV of global baseline BFI was 12(5)\%, whereas intermouse COV was 26(7)\% (Fig. 4, Table 2).
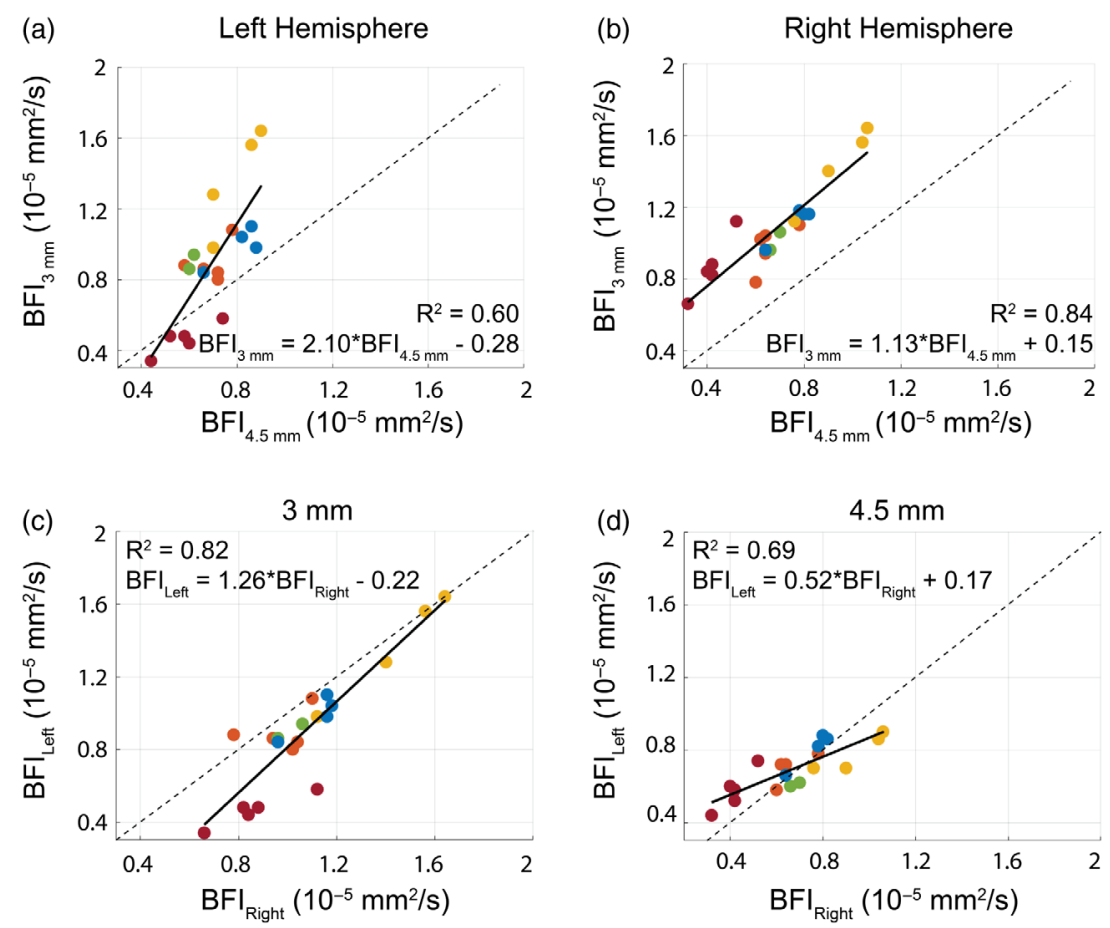

Fig. 3 Resting-state BFI. Resting-state $\mathrm{BFI}$ at 3 versus $4.5 \mathrm{~mm}\left(\mathrm{BFI}_{3 \mathrm{~mm}}\right.$ and $\mathrm{BFI}_{4.5 \mathrm{~mm}}$, respectively, in units of $10^{-5} \mathrm{~mm}^{2} / \mathrm{s}$ ) across all days, color coded by mouse, for (a) the left and (b) right hemispheres. Right versus left $\mathrm{BFI}\left(\mathrm{BFI}_{\text {right }}\right.$ and $\mathrm{BFI}_{\text {left }}$, respectively) across all days, color coded by mouse, for the (c) $3 \mathrm{~mm}$ and (d) $4.5 \mathrm{~mm}$ source-detector separations. The solid line in all subplots denotes the best-fit line to the data, whereas the dotted line reflects the line of unity. 
Table 1 Summary of correlations between detectors. Results of linear regression analysis quantifying the relationship of $\mathrm{BFI}, \mathrm{CVR}$, and $t_{1 / 2}$ across source-detector separations and hemispheres. Results are reported as slope and intercept [with associated confidence intervals $(\mathrm{Cl})$ ], along with the coefficient of determination, $R^{2}$, and $p$-value.

\begin{tabular}{llcccc}
\hline \hline & & Slope $(\mathrm{Cl})$ & Intercept $(\mathrm{Cl})$ & $R^{2}$ & $p$-value \\
\hline BFI & Left, 3 mm versus $4.5 \mathrm{~mm}$ & $2.10(1.91,2.29)$ & $-0.28(-0.35,-0.22) \times 10^{-5}$ & 0.60 & 0.07 \\
& Right, 3 mm versus $4.5 \mathrm{~mm}$ & $1.13(1.08,1.18)$ & $0.15(0.14,0.17) \times 10^{-5}$ & 0.84 & 0.001 \\
& 3 mm, left versus right & $1.26(1.19,1.32)$ & $-0.22(-0.26,-0.19) \times 10^{-5}$ & 0.82 & 0.001 \\
& $4.5 \mathrm{~mm}$, left versus right & $0.52(0.48,0.56)$ & $0.17(0.16,0.19) \times 10^{-5}$ & 0.69 & $<0.001$ \\
\hline CVR & 3 mm versus 4.5 mm & $1.00(1.00,1.02)$ & $-0.08(-2.74,2.56)$ & 0.99 & $<0.001$ \\
& Left versus Right & $1.00(0.99,1.01)$ & $-1.48(-2.18,-0.82)$ & 0.99 & $<0.001$ \\
\hline$t_{1 / 2}$ & 3 mm versus 4.5 mm & $0.90(0.88,0.92)$ & $0.87(-0.07,1.81)$ & 0.97 & $<0.001$ \\
& Left versus right & $0.99(0.98,0.99)$ & $1.97(1.60,2.34)$ & 0.99 & $<0.001$ \\
\hline \hline
\end{tabular}
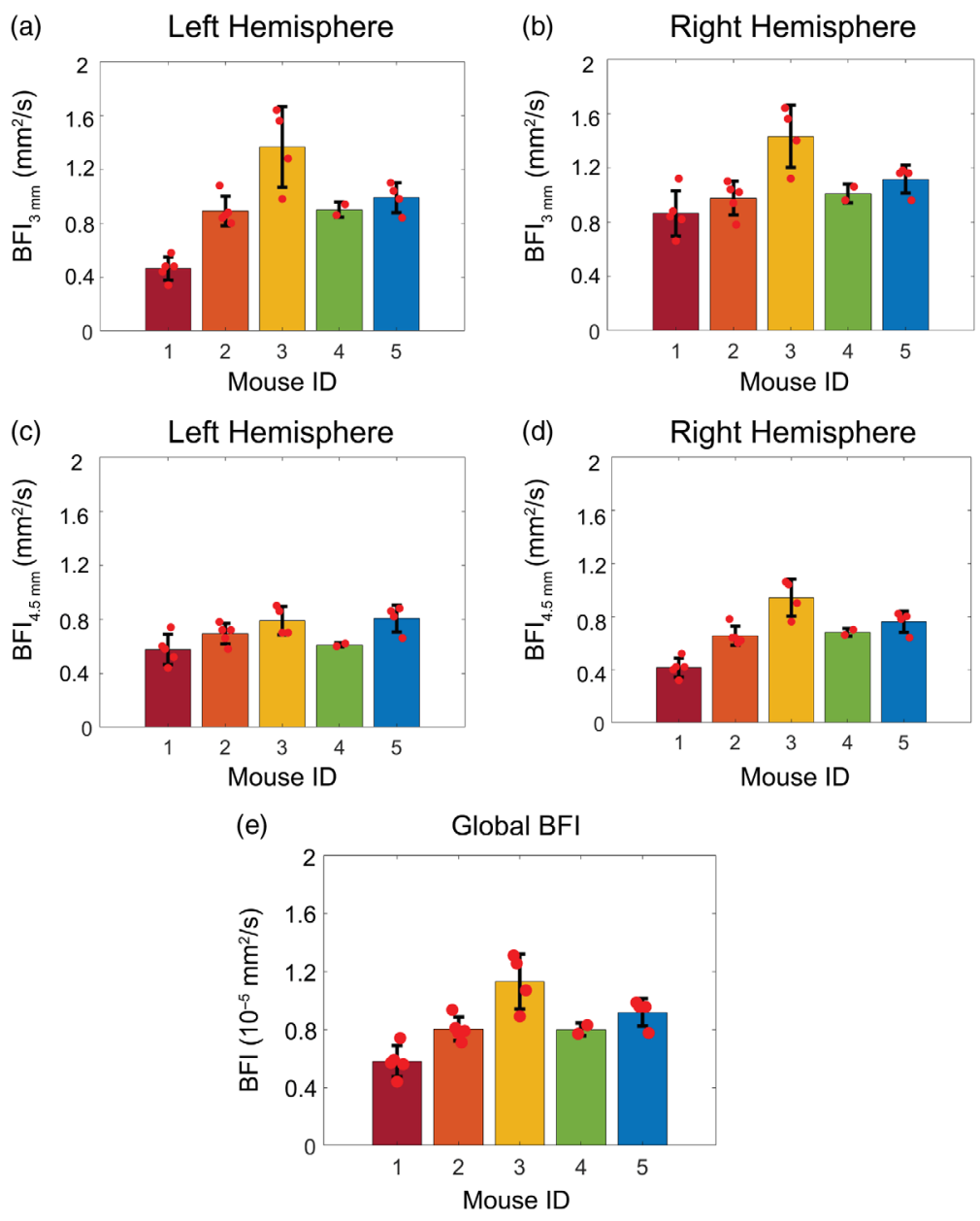

Fig. 4 Resting state BFI: (a)-(d) barplot of the mean baseline BFI values for each mouse at each detector and (e) barplot of the mean global baseline BFI values for each mouse. Red dots denote daily values and the black bars denote the standard deviation in the mean across all days measured. 
Table 2 Measurement repeatability. Mean \pm standard deviation coefficient of variation within mice $\left(\mathrm{COV}_{\text {intra }}\right)$ and between mice $\left(\mathrm{COV}_{\text {inter }}\right)$ for global measurements of baseline BFI, CVR, and response half-times $\left(t_{\frac{1}{2}}\right)$.

\begin{tabular}{lccc}
\hline \hline & Baseline BFI (\%) & CVR (\%) & $t_{\frac{1}{2}}(\%)$ \\
\hline COV $_{\text {intra }}$ & $12 \pm 5$ & $15 \pm 9$ & $24 \pm 15$ \\
COV $_{\text {inter }}$ & $26 \pm 7$ & $19 \pm 10$ & $27 \pm 11$ \\
\hline \hline
\end{tabular}
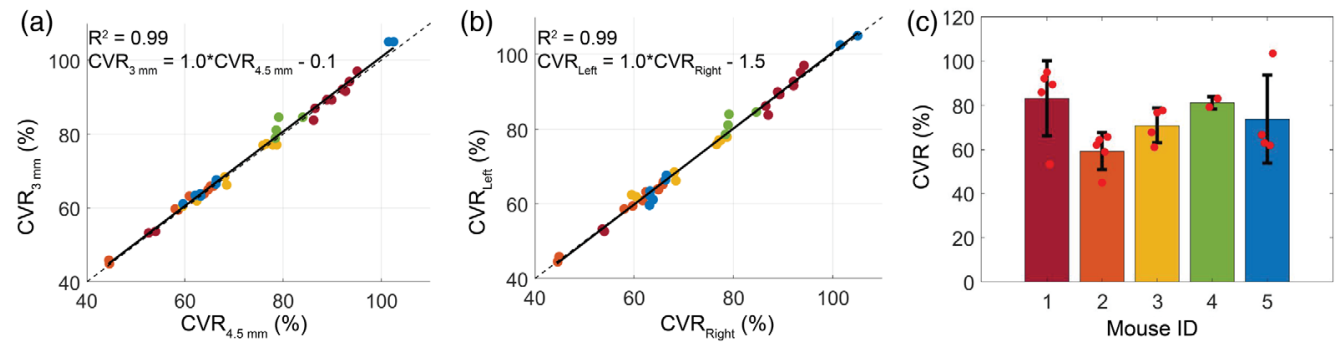

Fig. 5 Cerebrovascular reactivity. (a) CVR at $3 \mathrm{~mm}$ versus $4.5 \mathrm{~mm}\left(\mathrm{CVR}_{3 \mathrm{~mm}}\right.$ and CVR $4.5 \mathrm{~mm}$, respectively) across all days and both hemispheres, color coded by mouse. (b) CVR in the left versus right hemispheres $\left(\mathrm{CVR}_{\text {left }}\right.$ and $\mathrm{CVR}_{\text {right }}$, respectively) across all days and both sourcedetector separations, color coded by mouse. In both (a) and (b), the solid line in all subplots denotes the best-fit line to the data, whereas the dotted line reflects the line of unity. (c) Barplot of the mean global CVR for each mouse. Red dots denote daily values, and the black bars denote the standard deviation in the mean across days.

Although resting-state BFI differed significantly between source-detector separations and between hemispheres, CVR was remarkably similar ( $p>0.05$ for all paired comparisons). CVR was strongly correlated across source-detector separations [Fig. 5(a), Table 1]. Similarly, strong correlation was observed across hemispheres [Fig. 5(b), Table 1]. Mean(std) global CVR across all mice and all days of study was $73(15) \%$. The mean(std) intramouse COV of global CVR was 15(9.0)\%, whereas the intermouse COV was 19(10)\% [Fig. 5(c), Table 2].

Similar to CVR, the response half time $\left(t_{1 / 2}\right)$ to ACZ was also highly correlated across source-detector separations [Fig. 6(a), Table 1] and hemispheres [Fig. 6(b), Table 1]. Mean(std) global $t_{1 / 2}$ across all mice and all days of study was 70(22) s. Mean(std) intramouse COV for global $t_{1 / 2}$ was 24(15)\%, whereas intermouse COV was 27(11)\% [Fig. 6(c), Table 2].
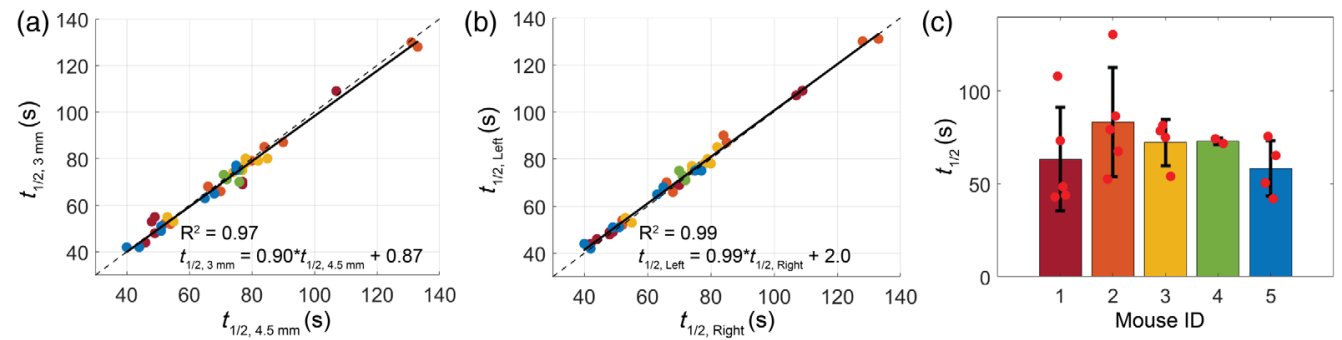

Fig. 6 Response half-time. (a) ACZ response half time at $3 \mathrm{~mm}$ versus $4.5 \mathrm{~mm}\left(t_{1 / 2,3 \mathrm{~mm}}\right.$ and $t_{1 / 2,4.5 \mathrm{~mm}}$, respectively) across all days and both hemispheres, color coded by mouse. (b) Response half-time in the left versus right hemispheres $\left(t_{1 / 2 \text {,left }}\right.$ and $t_{1 / 2 \text {,right }}$, respectively) across all days and both source-detector separations, color coded by mouse. In both (a) and (b), the solid line in all subplots denotes the best-fit line to the data, whereas the dotted line reflects the line of unity. (c) Bar plot of the mean global response time for each mouse. Red dots denote daily values and the black bar denotes the standard deviation in the mean across days. 


\section{Discussion}

Herein, we present an approach to estimate CVR in awake, free-behaving mice via intraperitoneal injection of ACZ coupled with minimally invasive assessment of cerebral blood flow with DCS. ACZ acts to inhibit carbonic anhydrase, an enzyme that aids in the transport of carbon dioxide from the tissues to the lungs. This inhibition increases local carbon dioxide levels by slowing carbon dioxide transport from the tissue to the blood stream, causing an increase in blood flow. ${ }^{53}$ Although intravenous ACZ is commonly used in humans to assess CVR, ${ }^{54}$ it is less common in mice due to difficulties with drug administration (i.e., tail vein injections require restraint acclimation, multiple injections for longitudinal studies create a risk for infection or vein collapse). Herein, we utilize intraperitoneal injection of ACZ to overcome these difficulties. Although drug uptake is slower and sometimes more variable with intraperitoneal versus intravenous injection, ${ }^{55}$ the relative ease of administration provides an obvious advantage.

To characterize this new technique, we quantified CVR in multiple mice across several days. We found that CVR and the response half-time were remarkably similar across hemispheres and across source-detector separations (Figs. 3 and 5), suggesting a homogenous, whole-brain response to ACZ. The homogeneity of this response in mice is in contrast to previous results in humans that show regional differences in brain response to ACZ. ${ }^{56} \mathrm{We}$ also characterized the inter- and intramouse CVR response (15\% and 19\%, respectively, Fig. 4). For context, CVR impairments $>25 \%$ have been reported for humans with internal carotid artery occlusions, ${ }^{59,60}$ white matter disease, ${ }^{60,61}$ stroke, ${ }^{9,60}$ and traumatic brain injury. ${ }^{23,62}$ Given the reported variability of our method, we should be sensitive to impairments of this magnitude. Our mean intersubject $\mathrm{COV}$ agrees with previous results in mice ${ }^{57}$ and is notably less than intersubject variability seen in humans with ACZ. ${ }^{58}$ We note that one source of variability with this approach is the use of intraperitoneal injection, which entails hepatic metabolism before ACZ enters the systemic circulation and reaches the brain. Differences in metabolism both between mice and across days may cause variability in ACZ response. ${ }^{55}$ In sum, we found this method of measuring CVR to be repeatable without the risks associated with multiple tail vein injections or the confounding influences of anesthesia.

This approach also enabled us to characterize resting-state blood flow in awake, freebehaving mice. Significant temporal variability $(\sim 20 \%)$ was observed during the 10-min baseline period that was likely physiological in origin, as motion artifacts were minimal and fluctuations were observed even during periods of inactivity. These variations may be attributed to uncontrolled fluctuations in neural activity, blood pressure, cardiac pulsatility, and/or carbon dioxide tension as the mouse ambulates freely. ${ }^{63}$ With the recent development of high-speed software correlators, ${ }^{64}$ future work may incorporate high-speed $(>100 \mathrm{~Hz})$ DCS acquisition to visualize pulsatile flow and better understand these temporal variations.

As expected, we found that the mean resting state blood flow was systematically lower at 4.5$\mathrm{mm}$ than 3-mm source-detector separations given the higher fraction of detected photons at the 4.5-mm separation that penetrate to the deeper, less-vascularized white matter wherein perfusion is lower. ${ }^{65,66}$ Interestingly, we also observed small, non-significant hemispheric differences in resting state blood flow. The cause of this hemispheric asymmetry is unknown, but regional perfusion asymmetries have also been observed in humans, ${ }^{67,68}$ and we have also observed similar asymmetries in unpublished results from other strains of mice measured in our lab. Average resting state global blood flow was relatively stable across multiple days of measurement [mean(std) intramouse $\mathrm{COV}=12(5) \%$ ]. However, substantial variation was observed across mice, in agreement with previous publications. ${ }^{69,70}$ Intramouse variation may arise, to some extent, because we have assumed fixed values for the absorption and reduced scattering coefficient across all mice in our estimation of BFI. Assuming $~ 10 \%$ variation in absorption and reduced scattering coefficients across mice, ${ }^{51}$ fixing these coefficients to assumed values may lead to errors in BFI on the order of $\sim 10 \%{ }^{71}$ Future work would benefit from simultaneous estimation of BFI and tissue optical properties.

Limitations of this method include a somewhat time-intensive surgery ( $\sim 45 \mathrm{~min}$ ) and recovery/acclimation period ( $\sim 3$ days), along with administration of a vasoactive substance that increases cerebral perfusion for up to $2 \mathrm{~h}$ postinjection (Video S1). We also note that repeated ACZ administration has been documented to increase production of carbonic anhydrase. ${ }^{72}$ 
This increase may cause mice to develop drug tolerance and thus may artificially attenuate the $\mathrm{CBF}$ response over time. However, we did not observe a decreased response to ACZ over the 1 -week time frame of our study. An additional limitation of this method is the assumption that tissue optical properties (i.e., the absorption and reduced scattering coefficients) do not change appreciably in response to ACZ. Recent work in rats has shown that optical properties do not change during hypercapnia; ${ }^{73}$ however, the effect of $\mathrm{ACZ}$ on optical property changes is unknown. Given that ACZ and hypercapnia act via similar mechanisms, i.e., to increase carbon dioxide tension in the tissue, we believe this assumption to be a suitable first-order approximation. If false, a $10 \%$ increase in the reduced scattering coefficient could lead to errors in rCBF of $\sim 20 \%$. ${ }^{44}$ Thus as mentioned above, future studies would benefit greatly from the use of frequency domain near-infrared spectroscopy ${ }^{51}$ or multidistance $\mathrm{DCS}^{74}$ to continuously quantify absorption and reduced scattering coefficients.

Finally, we comment on the relatively high rate that data were discarded in this study (20\%). Three out of five discarded datasets were rejected from a single mouse due to poor sensor contact with the optical window as a result of dried dental dement on the window surface. Future studies should employ careful visual inspection of the window surface during surgery to remove any surface imperfections that prohibit proper sensor contact with the window. The remaining two discarded datasets were rejected due to abrupt movement during ACZ injection that caused the needle (and thus the ACZ solution) to become displaced from its intended target of the intraperitoneal cavity. This motion occurred in two separate mice toward the end of the study (days 4 and 5) and was possibly caused by skin soreness after multiple days of repeated injections. If so, a topical analgesic may mitigate this issue when repeated injections are warranted.

\section{Conclusions}

This study reports a method for longitudinal assessments of cerebral blood flow and CVR in awake, free-behaving mice which can be easily adapted to assess cerebrovascular impairment in various disease states. This approach overcomes several key challenges of vascular reactivity assessment in mice by removing the need for a repeated tail vein injection, femoral arterial catheter, intubation, or the confounding influence of anesthesia. Future work can utilize this method to screen for cerebrovascular impairments in murine models of disease and to track changes in vascular reactivity with therapeutic intervention.

\section{Disclosures}

The authors have no conflicts of interest.

\section{Acknowledgments}

This work would not have been possible without the help of Dr. Adam Bauer and Annie Bice. This work was supported by the National Institutes of Health (Nos. R21 NS104801 and R01 NS115994) (EMB) and the Children's Healthcare of Atlanta Junior Faculty Focused Award (EMB). This material is based upon work supported by the National Science Foundation Graduate Research Fellowship Program under Grant No. 1937971. Any opinions, findings, and conclusions or recommendations expressed in this material are those of the authors and do not necessarily reflect the views of the National Science Foundation.

\section{References}

1. P. Liu, J. B. De Vis, and H. Lu, "Cerebrovascular reactivity (CVR) MRI with $\mathrm{CO}_{2}$ challenge: a technical review," NeuroImage 187, 104-115 (2019).

2. J. B. De Vis et al., "Calibrated MRI to evaluate cerebral hemodynamics in patients with an internal carotid artery occlusion," J. Cereb. Blood Flow Metab. 35(6), 1015-1023 (2015). 
3. M. J. Donahue et al., "Relationships between hypercarbic reactivity, cerebral blood flow, and arterial circulation times in patients with moyamoya disease: vascular reactivity in moyamoya disease," J. Magn. Reson. Imaging 38(5), 1129-1139 (2013).

4. A. Gupta et al., "Cerebrovascular reserve and stroke risk in patients with carotid stenosis or occlusion: a systematic review and meta-analysis," Stroke 43(11), 2884-2891 (2012).

5. D. M. Mandell et al., "Mapping cerebrovascular reactivity using blood oxygen leveldependent MRI in patients with arterial steno-occlusive disease: comparison with arterial spin labeling MRI," Stroke 39(7), 2021-2028 (2008).

6. D. J. Mikulis et al., "Preoperative and postoperative mapping of cerebrovascular reactivity in moyamoya disease by using blood oxygen level—dependent magnetic resonance imaging," J. Neurosurg. 103(2), 347-355 (2005).

7. J. R. Marstrand et al., "Cerebral perfusion and cerebrovascular reactivity are reduced in white matter hyperintensities," Stroke 33(4), 972-976 (2002).

8. Y. Fujiwara et al., "Simultaneous impairment of intracranial and peripheral artery vasoreactivity in CADASIL patients," Cerebrovasc. Diseases 33(2), 128-134 (2012).

9. F. Geranmayeh et al., "Measuring vascular reactivity with breath-holds after stroke: a method to aid interpretation of group-level BOLD signal changes in longitudinal fMRI studies," Hum. Brain Mapp. 36(5), 1755-1771 (2015).

10. K. Alexandre et al., "Regional impairment of cerebrovascular reactivity and BOLD signal in adults after stroke," Stroke 36(6), 1146-1152 (2005).

11. S. Mauro et al., "Transcranial Doppler assessment of cerebrovascular reactivity in symptomatic and asymptomatic severe carotid stenosis," Stroke 27(11), 1970-1973 (1996).

12. M. W. Webster et al., "Compromised cerebral blood flow reactivity is a predictor of stroke in patients with symptomatic carotid artery occlusive disease," J. Vasc. Surg. 21(2), 338-345 (1995).

13. T.-Y. Chang et al., "Change in cerebral perfusion after carotid angioplasty with stenting is related to cerebral vasoreactivity: a study using dynamic susceptibility-weighted contrastenhanced MR imaging and functional MR imaging with a breath-holding paradigm," Am. J. Neuroradiol. 30(7), 1330-1336 (2009).

14. C. Iadecola and R. L. Davisson, "Hypertension and cerebrovascular dysfunction," Cell Metab. 7(6), 476-484 (2008).

15. J. K. Lee et al., "A pilot study of cerebrovascular reactivity autoregulation after pediatric cardiac arrest," Resuscitation 85(10), 1387-1393 (2014).

16. F. Mathieu et al., "Continuous near-infrared spectroscopy monitoring in adult traumatic brain injury: a systematic review," J. Neurosurg. Anesthesiol. 32(4), 288-299 (2020).

17. F. A. Zeiler et al., "Intracranial and extracranial injury burden as drivers of impaired cerebrovascular reactivity in traumatic brain injury," J. Neurotrauma 35(14), 1569-1577 (2018).

18. F. A. Zeiler et al., "Critical thresholds of intracranial pressure-derived continuous cerebrovascular reactivity indices for outcome prediction in noncraniectomized patients with traumatic brain injury," J. Neurotrauma 35(10), 1107-1115 (2018).

19. K. Kenney et al., "Cerebral vascular injury in traumatic brain injury," Exp. Neurol. 275, 353-366 (2016).

20. E. Tchistiakova et al., "Combined effects of type 2 diabetes and hypertension associated with cortical thinning and impaired cerebrovascular reactivity relative to hypertension alone in older adults," Neurolmage 5, 36-41 (2014).

21. J. Buterbaugh et al., "Cerebrovascular reactivity in young subjects with sleep apnea," Sleep 38(2), 241-250 (2015).

22. D. O. Svaldi et al., "Cerebrovascular reactivity alterations in asymptomatic high school football players," Dev. Neuropsychol. 40(2), 80-84 (2015).

23. D. M. Bailey et al., "Impaired cerebral haemodynamic function associated with chronic traumatic brain injury in professional boxers," Clin. Sci. 124(3), 177-189 (2013).

24. D. O. Svaldi et al., "Cerebrovascular reactivity changes in asymptomatic female athletes attributable to high school soccer participation," Brain Imaging Behav. 11(1), 98-112 (2017). 
25. S. C. Rose et al., "Neurocognitive function and head impact burden over two seasons of youth tackle football," J. Neurotrauma 36(19), 2803-2809 (2019).

26. E. V. Groman and C. P. Reinhardt, "Method to quantify tail vein injection technique in small animals," Contemp. Top. Lab. Anim. Sci. 43(1), 35-38 (2004).

27. P. Lacombe et al., "Impaired cerebral vasoreactivity in a transgenic mouse model of cerebral autosomal dominant arteriopathy with subcortical infarcts and leukoencephalopathy arteriopathy," Stroke 36(5), 1053-1058 (2005).

28. J. Silva et al., "Transient hypercapnia reveals an underlying cerebrovascular pathology in a murine model for HIV-1 associated neuroinflammation: role of NO-cGMP signaling and normalization by inhibition of cyclic nucleotide phosphodiesterase-5," J. Neuroinflammation 9(1), 253 (2012).

29. A. Y. Lai et al., "Venular degeneration leads to vascular dysfunction in a transgenic model of Alzheimer's disease," Brain 138(4), 1046-1058 (2015).

30. A. Dorr et al., "Amyloid- $\beta$-dependent compromise of microvascular structure and function in a model of Alzheimer's disease," Brain 135(10), 3039-3050 (2012).

31. J. A. Wells et al., "Increased cerebral vascular reactivity in the tau expressing $\mathrm{rTg} 4510$ mouse: evidence against the role of tau pathology to impair vascular health in Alzheimer's disease," J. Cereb. Blood Flow Metab. 35(3), 359-362 (2015).

32. L. Park et al., "Nox $x_{2}$-derived radicals contribute to neurovascular and behavioral dysfunction in mice overexpressing the amyloid precursor protein," Proc. Natl. Acad. Sci. U. S. A. 105(4), 1347-1352 (2008).

33. E. Sathialingam et al., "Small separation diffuse correlation spectroscopy for measurement of cerebral blood flow in rodents," Biomed. Opt. Express 9(11), 5719 (2018).

34. D. A. Boas and A. G. Yodh, "Spatially varying dynamical properties of turbid media probed with diffusing temporal light correlation," J. Opt. Soc. Am. A 14(1), 192 (1997).

35. D. A. Boas, L. E. Campbell, and A. G. Yodh, "Scattering and imaging with diffusing temporal field correlations," Phys. Rev. Lett. 75(9), 1855-1858 (1995).

36. T. Durduran et al., "Diffuse optics for tissue monitoring and tomography," Rep. Prog. Phys. 73(7), 076701 (2010).

37. T. Durduran and A. G. Yodh, "Diffuse correlation spectroscopy for non-invasive, microvascular cerebral blood flow measurement," NeuroImage 85, 51-63 (2014).

38. T. Durduran, "Noninvasive measurements of tissue hemodynamics with hybrid diffuse optical methods," Med. Phys. 31(7), 2178-2178 (2004).

39. R. C. Mesquita et al., "Direct measurement of tissue blood flow and metabolism with diffuse optics," Philos. Trans. R. Soc. A 369(1955), 4390-4406 (2011).

40. E. M. Buckley et al., "Monitoring hemodynamic changes in preterm infants using optical spectroscopies and Doppler ultrasound," in Biomed. Opt., p. BTuF35, OSA, St. Petersburg, Florida (2008).

41. E. M. Buckley et al., "Cerebral hemodynamics in preterm infants during positional intervention measured with diffuse correlation spectroscopy and transcranial Doppler ultrasound," Opt. Express 17(15), 12571 (2009).

42. C. Menon et al., "An integrated approach to measuring tumor oxygen status using human melanoma xenografts as a model," Cancer Res. 63(21), 7232-7240 (2003).

43. N. Roche-Labarbe et al., "Noninvasive optical measures of $\mathrm{CBV}, \mathrm{StO}_{2}, \mathrm{CBF}$ index, and $\mathrm{rCMRO}_{2}$ in human premature neonates' brains in the first six weeks of life," Hum. Brain Mapp. 31(3), 341-352 (2010).

44. E. M. Buckley et al., "Validation of diffuse correlation spectroscopic measurement of cerebral blood flow using phase-encoded velocity mapping magnetic resonance imaging," J. Biomed. Opt. 17(3), 037007 (2012).

45. S. A. Carp et al., "Validation of diffuse correlation spectroscopy measurements of rodent cerebral blood flow with simultaneous arterial spin labeling MRI; towards MRIoptical continuous cerebral metabolic monitoring," Biomed. Opt. Express 1(2), 553 (2010).

46. M. N. Kim et al., "Noninvasive measurement of cerebral blood flow and blood oxygenation using near-infrared and diffuse correlation spectroscopies in critically brain-injured adults," Neurocrit. Care 12(2), 173-180 (2010). 
47. M. Diop et al., "Calibration of diffuse correlation spectroscopy with a time-resolved nearinfrared technique to yield absolute cerebral blood flow measurements," Biomed. Opt. Express 2(7), 2068 (2011).

48. C. Zhou et al., "Diffuse optical monitoring of hemodynamic changes in piglet brain with closed head injury," J. Biomed. Opt. 14(3), 034015 (2009).

49. Correlator.com, www.correlator.com.

50. S. Y. Lee et al., "Noninvasive optical assessment of resting-state cerebral blood flow in children with sickle cell disease," Neurophotonics 6(3), 035006 (2019).

51. S. Y. Lee et al., "Small separation frequency-domain near-infrared spectroscopy for the recovery of tissue optical properties at millimeter depths," Biomed. Opt. Express 10(10), 5362-5377 (2019).

52. MathWorks Inc., "MATLAB," The MathWorks Inc., Natick, Massachusetts (2020).

53. W. H. Freeman, Biochemistry, W.H. Freeman Publishers, New York (2002).

54. A. S. Vagal et al., "The acetazolamide challenge: techniques and applications in the evaluation of chronic cerebral ischemia," Am. J. Neuroradiology 30(5), 876-884 (2009).

55. P. V. Turner et al., "Administration of substances to laboratory animals: routes of administration and factors to consider," J. Am. Assoc. Lab. Anim. Sci. 50(5), 600-613 (2011).

56. C. Grandin et al., "Absolute CBF and CBV measurements by MRI bolus tracking before and after acetazolamide challenge: repeatabilily and comparison with PET in humans," NeuroImage 26(2), 525-535 (2005).

57. N. Hecht et al., "Cerebral hemodynamic reserve and vascular remodeling in C57/BL6 mice are influenced by age," Stroke 43(11), 3052-3062 (2012).

58. E. Højer-Pedersen, "Effect of acetazolamide on cerebral blood flow in subacute and chronic cerebrovascular disease," Stroke 18(5), 887-891 (1987).

59. R. P. H. Bokkers et al., "Cerebrovascular reactivity within perfusion territories in patients with an internal carotid artery occlusion," J. Neurol. Neurosurg. Psychiatry 82(9), 1011-1016 (2011).

60. D. P. J. Smeeing et al., "Arterial spin labeling and blood oxygen level-dependent MRI cerebrovascular reactivity in cerebrovascular disease: a systematic review and metaanalysis," Cerebrovasc. Diseases 42(3-4), 288-307 (2016).

61. K. Sam et al., "Impaired dynamic cerebrovascular response to hypercapnia predicts development of white matter hyperintensities," NeuroImage 11, 796-801 (2016).

62. E. Sorrentino et al., "Critical thresholds for cerebrovascular reactivity after traumatic brain injury," Neurocrit. Care 16(2), 258-266 (2012).

63. S. Vorstrup, O. B. Paulson, and N. A. Lassen, "Cerebral blood flow in acute and chronic ischemic stroke using xenon-133 inhalation tomography," Acta Neurol. Scand. 74(6), 439451 (1986).

64. D. Wang et al., "Fast blood flow monitoring in deep tissues with real-time software correlators," Biomed. Opt. Express 7(3), 776-797 (2016).

65. L. M. Parkes et al., "Normal cerebral perfusion measurements using arterial spin labeling: reproducibility, stability, and age and gender effects," Magn. Reson. Med. 51(4), 736-743 (2004).

66. T. J. Carroll et al., "Quantification of cerebral perfusion using the 'bookend technique': an evaluation in CNS tumors," Magn. Reson. Imaging 26(10), 1352-1359 (2008).

67. X. Guo et al., "Asymmetry of cerebral blood flow measured with three-dimensional pseudocontinuous arterial spin-labeling MR imaging in temporal lobe epilepsy with and without mesial temporal sclerosis: asymmetry of CBF in TLE patients," J. Magn. Reson. Imaging 42(5), 1386-1397 (2015).

68. J. S. Perlmutter et al., "Regional asymmetries of cerebral blood flow, blood volume, and oxygen utilization and extraction in normal subjects," J. Cereb. Blood Flow Metab. 7(1), 64-67 (1987).

69. E. M. Buckley et al., "Decreased microvascular cerebral blood flow assessed by diffuse correlation spectroscopy after repetitive concussions in mice," J. Cereb. Blood Flow Metab. 35(12), 1995-2000 (2015).

70. S. B. Sankar et al., "Low cerebral blood flow is a non-invasive biomarker of neuroinflammation after repetitive mild traumatic brain injury," Neurobiol. Disease 124, 544-554 (2019). 
71. D. Irwin et al., "Influences of tissue absorption and scattering on diffuse correlation spectroscopy blood flow measurements," Biomed. Opt. Express 2(7), 1969-1985 (2011).

72. F. L. Engstrom et al., "Acute and chronic acetazolamide administration in DBA and C57 mice: effects of age," Epilepsia 27(1), 19-26 (1986).

73. J. Sutin et al., "Time-domain diffuse correlation spectroscopy," Optica 3(9), 1006 (2016).

74. P. Farzam and T. Durduran, "Multidistance diffuse correlation spectroscopy for simultaneous estimation of blood flow index and optical properties," J. Biomed. Opt. 20(5), 055001 (2015).

Rowan O. Brothers is a doctoral student and NSF graduate research fellow in biomedical engineering at Georgia Tech and Emory University. He received his bachelor's degree in chemistry from Georgia State University in May 2017. His current research focuses on acute cerebral hemodynamics as potential biomarkers of cognitive and pathological outcome in a pre-clinical murine model of mild traumatic brain injury.

Nir Atlas, MD, is a fellow of pediatric critical care medicine at Children's Healthcare of Atlanta, Emory University. His interests in biomedical innovation, coupled with neuro and cardiac critical care, have spawned clinical and preclinical projects with Dr. Erin Buckley at Emory University/ Georgia Tech Department of Biomedical Engineering. With his research, Dr. Atlas aims to form collaborative partnerships with leaders in biomedical technologies and therapeutics to improve pediatric neuro protective strategies and outcomes.

Kyle R. Cowdrick is a biomedical engineering doctoral candidate at Georgia Tech and Emory University. He received his master's in bioengineering innovation and design at The Johns Hopkins University as a founding member of SpineAlign Surgical LLC. Kyle also serves as the New Partnerships Liaison for Georgia Tech's BME Capstone design program. His research focuses on early-stage clinical feasibility studies to assess the translational potential of diffuse optics in pediatric cardiac arrest and traumatic brain injury.

Erin M. Buckley is an assistant professor in the Wallace H. Coulter Department of Biomedical Engineering at Georgia Institute of Technology/Emory University and the Department of Pediatrics at Emory University. She received her PhD from the University of Pennsylvania's Department of Physics and Astronomy in 2011, and completed postdoctoral training at the Children's Hospital of Philadelphia and at Massachusetts General Hospital. Her research focuses on the development diffuse optical spectroscopies for brain monitoring. 